\title{
Both for Ethics and Health. Non-Animal Technologies: an achievable goal
}

\section{Celentano ${ }^{1}$}

${ }^{1}$ Dipartimento di Lettere e Filosofia. Università degli Studi di Cassino e del Lazio Meridionale. Via Zamosch, 43 03043 Cassino - Italy.

submitted: May 12, 2017, accepted: May 13, 2017, EPub Ahead of Print: May 27, 2017

Conflict of interest: None

DOI: 10.24019/jtavr.21 - Corresponding author: Prof. Marco Celentano, marcelen@unina.it

(C) 2017 Fondazione Vasculab impresa sociale ONLUS. All rights reserved.

\begin{abstract}
The international debate on animal testing, its improvement through the well known "3Rs" methodology, and the possibility of its replacement with the emergent NATs (Non-Animal Technologies) brought into the new millennium a turn. Nations as U.S.A. and U.K., and partly international bodies as the EU. seem be doing a renewed effort to diminish and possibly replace animal testing with more reliable and less invasive techniques, to which also the giants of the chemical and pharmaceutical industry seem very interested.
\end{abstract}

Keywords Animal Testing, Non-animal Technologies (NATs), Toxicogenomics, 3Rs, Ethics.

During the $20^{\text {th }}$ century, animals were used in a large range of scientific areas: basic biological, medical and veterinary research, pharmacology and dentistry, environmental monitoring and toxicology, testing of ballistic, chemical, nuclear and biological weapons, as well as in neuroscience and experimental psychology.

However, the international debate on animal testing, its "improvement" (the well known "3Rs") ${ }^{[\mathrm{i}]} 1$, and its replacement with the emergent NATs (Non Animal Technologies) brought a turn into the new millennium. A change of strategies which consist of a renewed effort to diminish and possibly completely replace animal testing with more reliable and less invasive techniques, and which regards both the control bodies and research institutions of nations as U.S.A. and U.K., or supranational organizations as the European Union, and the giants of the chemical, agrochemical, pharmaceutical, medical and veterinary industry.

What the reasons of this change? At least three motives emerge from the on-going debate:

- $\quad$ the undeniable evidence of the poor predictive value of animal experimentation in crucial fields as toxicology and pharmacology (according to PubMed and the U.S. Food and Drug Administration, 92\% of the drugs harmless in non-human animals are then discarded during the mandatory clinical trials in humans);

- the damage to health caused by it (the most well-known and severe case was that of Talidomide, a tranquilizer for pregnant women that, harmless in animal tests, turned out to be teratogenic to our species, but was withdrawn only when more than 10,000 phocomelics babies were born);

- the advent of in vitro cellular technologies and in silico (computational) methods, which turned out to be more reliable, economical and faster than animal testing.

An important step in this turn took place in 2007, with the publication of the results of the study Toxicity Testing in the 21st Century: a Vision and a Strategy, commissioned by the U.S. Environmental Protection Agency (EPA) in order to promote an innovative approach to toxicity testing, and edited by the staff of Committee on Toxicity Testing and Assessment of Environmental Agents of the National Research Council (NRC), which "includes experts in developmental toxicology, reproductive toxicology, 
neurotoxicology, immunology, pediatrics and neonatology, epidemiology, biostatistics, in vitro methods and models, molecular biology, pharmacology, physiologically based pharmacokinetic and pharmacodynamic models, genetics, toxicogenomics, cancer hazard assessment, and risk assessment" (Krewski et al. 2010) ${ }^{[\mathrm{ii}]} 2$.

What the EPA asked to the NRC was a strategy which, over the next few decades, could be able to turn the research in toxicology from the current expensive and lengthy in vivo testing to in vitro and in silico technologies in which the "assays will be conducted primarily with cells or cell lines, optimally with human cells or cell lines, and as time passes, the need for traditional apical animal tests will be greatly reduced and optimally eliminated" ${ }^{[i i i]}$ (Ivi).

Which the results? According to the authors, thanks to the introduction of toxicogenomics (or cellular toxicology), based on the analysis of the reactions of genes contained in human cells or cell lines tested to the chemicals, a "revolution is taking place in biology. At its centre is the progress being made in the elucidation of cellular-response networks. Those networks are interconnected pathways composed of complex biochemical interactions of genes, proteins, and small molecules that maintain normal cellular function, control communication between cells, and allow cells to adapt to changes in their environment. A familiar cellular-response network is signalling by estrogens in which initial exposure results in enhanced cell proliferation and growth of specific tissues or in proliferation of estrogen-sensitive cells in culture [...]. In that type of network, initial interactions between a signalling molecule and various cellular receptors result in a cascade of early, midterm, and late responses to achieve a coordinated response that orchestrates normal physiologic functions ". (Ivi)

What do the authors forecast about the future of animal and non-animal testing? The committee envisions a future in which, if a sufficient effort will be made, tests based on human cell systems will be able to replace, completely or almost totally, testing in animals.

After the first publication of this study in the US followed a series of experiments with the new Comp.Tox (Computational Toxicology), based on the use of "robots" that allow an assessment of the toxicity of chemicals at high speed. "The program, initially started at EPA as Tox.Cast to assess 1,000 chemicals (and known as Tox21 in its expanded form), employs a robot to speed chemical screening" which "wells replace the old standby of toxicology-animal testing. In addition to being slow and controversial, animal tests do not reveal how a chemical might impact humans, nor do they deliver any insight into the mechanisms by which a given chemical produced toxic outcomes. Simply by running the robotic tests, the EPA and its partner agencies will generate more information on chemical toxicity in the next few years than has been created in the past century"(Biello 2011) ${ }^{3}$. One of the most ambitious studies in the field is the Human Toxome Project which will comprehensively map the pathways of endocrine disruption (ED), as a first step towards mapping the whole human toxome.

If the new strategy outlined by the US EPA and NRC suggests a transition to end animal testing in more or less long times, but in fact still proposes procedures that include it, while reducing its use, the UK seems more clearly geared towards achieving this goal in just over a decade: by 2030 .

A significant testimony to this effort is the study "A non-animal technologies roadmap for the UK. Advancing predictive biology", published in November 2015 and drawn up by the governmental agency Innovate UK, the National Centre for the Replacement Refinement and Reduction of Animals in Research, the Biotechnology and Biological Sciences Research Council, the Defence, Science and Technology Laboratory, the Engineering and Physical Sciences Research Council and the Medical Research Council. It provides that, over the next thirteen / fourteen years, non-animal technologies (NATs) could potentially replace the use of animals, and intended "to guide the efforts of all those working in this area" (NC3Rs 2015 , p. 2$)^{4}$.

The study underscores in particular the enormous potential of development of this market sector and the huge amount of investment it already attracts. According to the authors, the "global market for cell based assays in drug discovery, safety, and toxicology will reach $\$ 21.6$ billion by 2018. The estimated global market for induced pluripotent stem cells is expected to reach $\$ 2.9$ billion in 2018, and the $3 \mathrm{D}$ cell culture market is expected to grow to about $\$ 2.2$ billion in 2019" (Ibidem, p. 4).

According to other estimates, published in BBC Research's 2014, "the only in vitro toxic test market was in $2012 \$ 4.9$ billion and will reach nearly 9.9 billion in 2017, with a compound annual growth rate of $14.7 \%$ for the five-year period 2012-2017" (translated from the Italian text) (Lucchini 2016) ${ }^{5}$. And these are figures that refer to the global market for in vitro tests but only in the field of toxicology, which should be added, for a realistic calculation, to "the value of what even the Kits and methods applied to research", as suggests G. Dal Negro, World-wide Director 3Rs at GlaxoSmithKline, interviewed in 2016 by C. Lucchini (Ivi).

But, what about the European Union? Here we find a strange situation: on one side, EU, adopting in 2004 the REACH (Registration, Evaluation and Authorization of Chemicals), a regulation designed to improve the protection of human health and environment from harmful chemicals, promoting methods alternative to animal testing, 
anticipated and inspired the American and English turn. On the other hand, some innovative guidelines suggested by REACH, according to which all chemicals placed on the market should be tested for their side effects on humans and the environment with the methods of emerging toxicogenomics (and with costs incurred by manufacturing industries), have had so far limited application and disappointing practical effects.

This is due to delays in the application of the new rules, insufficient investment, and the several exceptions to the constraints imposed on the use of animals in experiments, which REACH actually allows. It is in fact a document that promotes "alternative" methods to animal experimentation, meaning by this formula, not necessarily and not merely replacing techniques which do not imply animal testing, but also methods that, following the principle of the $3 \mathrm{Rs}$, decrease the number of animals employed or the suffering endured by them.

Not by chance, according to the Seventh Report on the Statistics on the number of animals used for experimental and other scientific purposes in the EU Member States, the results were so far relatively modest: in Europe the number of animals used for "scientific purposes" have gone from 12 million subjects used in laboratories by industry, universities and research centres in 2008, to 11.5 million in 2011. And only since 11 March 2013, in application of the Directive 2010/63/EU, it has entered into force in EU the ban on the marketing of cosmetics tested on animals.

\section{What is, in the end, the situation in Italy?}

Early in 2014, at request of the Environment Commissioner J. Potocnik, Italy was denounced at the European Court of Justice for failing to transpose the already mentioned Directive 63/2010, that imposes to improve the principle of the $3 \mathrm{Rs}$.

Italy replied approving on March 4, 2014 the Legislative Decree, no. 26 which, acknowledging the European Directive and in some ways even overtaking it, establishes inter alia that all research projects involving the use of vertebrate animals and certain invertebrates, such as Cephalopods, must be authorized by the Ministry of Health and carried out within authorized user establishments. But in February 2017 the application of the new legislation was set for a three-year period, for the research on abusers and xenografts.

Trying to take a snapshot of the current situation, we could say that in some areas, such as toxicology, pharmacology and environmental testing, new emergent NATs seem able to overcome and replace, in a few decades, the animal testing, with a political will that goes towards this and if different interests do not prevail. In vitro technologies and human cell cultures have proved to be more rigorous, faster and cheaper than animal testing in crucial fields as cancer-screening treatments, or drugs and environmental testing. In other areas, such as neurophysiology and the study of brain diseases or of the cardiovascular system, where it is more difficult to replace in vivo models with cell cultures or computerized simulations, given their enormous complexity, the goal seems farther and hard to reach but ${ }^{[i v] ~} 6,7$, also in this areas, some recent technical innovations have been very important. Very sophisticated non-invasive imaging methods such as the CT scan (computed tomography), the MRI (magnetic resonance imaging), the AMS (accelerator mass spectroscopy), the DTI (diffusion tensor imaging), or the MEG (magnetoencephalography) allow real-time measurements of associations between structure and function in humans with possible resolutions down to the single cells. In vitro models of the brain and of the blood-brain barrier (BBB) are today used for studies of neurotransmitter pathways, electrophysiological characteristics, morphological associations of human pathologies as Alzheimer's, Parkinson's, Huntington's diseases and epilepsy. They are today in use in cardiology computer models of the human heart which show the heart beat and heart reactions in $3 \mathrm{D}$; the recent development of microfluidics, based both on in vitro and in silico technologies, even though it is far from the ambitious goal of a hypothetical Lab-on-a-Chip, capable of concentrating in a millimeter chip all the operations needed for scientific testing, certainly represents a breakthrough.

In summary, is there reason to hope that, in a not too distant future, animal testing can be completely overcome or reduced to very rare limit cases? My view is that to achieve such goals technological innovations are never enough. It will be necessary to spread among scientists and people a critical awareness of all the ethical and medical implications of animal experimentation; a strong social and cultural commitment will be needed by those who are already today sensitive to these issues.

Both for Ethics and Health.

\section{Endnotes}

[i] Introduced in 1959 by WMS Russell and RL Burch (Russell 1959), of the Universities Federation of Animal Welfare, the principle suggests three guidelines for the scientific experimentation:

Replace the use of animals with alternative techniques, or avoid the use of animals altogether.

Reduce the number of animals used to a minimum, to obtain information from fewer animals or more information from the same number of animals.

Refine the way experiments are carried out, to make sure animals suffer as little as possible. This includes better housing and improvements to procedures which minimize pain and suffering and/or improve animal welfare.

[ii] See the part titled: "The Committee's First Task and Key Points From its Interim Report" in the updated version of the essay (Krewski et al.2010). 
[iii] See the part titled: "Component B: Toxicity Testing of Compounds and Metabolites".

[iv] For an introduction to the difficulties encountered by the project of a complete overcoming of animal testing, see Di Porzio (2012); Mario Negri Institute (2013).

\section{References}

1) Russel WMS, Burch RL. The Principles of Human Experimental Technique. London: Methuen \& Co. Ltd. 1959.

2) Krewski D, Acosta Jr D, Andersen M, Anderson H, Bailar III JC, Boekelheide K et al. (Staff of Committee on Toxicity Testing and Assessment of Environmental Agents).Toxicity Testing in the 21st Century: a Vision and a Strategy.Journal of Toxicology and Environmental Health, Health B: Critical Reviews. 2010 Feb 13; (0):51-138, available at: https://www.ncbi.nlm.nih.gov/pmc/articles/ PMC4410863/.
3) Biello D. Robot Allows High-Speed Testing of Chemicals. Scientific American. 13.10.11, available at: https://www.scientificamerican.com/article/robot-allows-highspeed-chemical-testing/

4) National Centre for the Replacement Refinement and Reduction of Animals in Research, Medical Research Council, Biotechnology and Biological Sciences Research Council, Defence, Science and Technology Laboratory, Engineering and Physical Sciences Research Council, Innovate UK. A non-animal technologies roadmap for the UK. Advancing predictive biology. 2015:2.

5) Lucchini C. Notiziario Chimico Farmaceutico. 2016 June; available at http://www.notiziariochimicofarmaceutico.it/ files/2016/12/Metodi-alternativi-alla-sperimentazione-animale.pdf.

6) Di Porzio U. Predatori dell'arca di Noé? In: Celentano M, de Mori B, Zecchinato P. Etologia ed Etica; Roma: Aracne; 2012. p. $155-62$.

7) Mario Negri Institute, Milan, Annual Report 2013; http:// www.marionegri.it/media/annual_report/en/rapportoricerca2013.pdf 